\title{
Habitat destruction and the extinction debt revisited: The Allee effect
}

\author{
Ling-ling Chen ${ }^{\mathrm{a}, *}$, Cang Hui ${ }^{\mathrm{b}}$ \\ a School of Humanities Science, JingLing Institute of Technology, Nanjing, Jiangsu 211169, China \\ ${ }^{\mathrm{b}}$ Centre for Invasion Biology, Department of Botany and Zoology, University of Stellenbosch, Private Bag X1, Matieland 7602, South Africa
}

\section{A R T I C L E I N F O}

\section{Article history:}

Received 18 November 2008

Received in revised form 4 June 2009

Accepted 21 June 2009

Available online 25 June 2009

\section{Keywords:}

Habitat destruction

Extinction debt

Allee effect

Time lag

Biodiversity

\begin{abstract}
A B S T R A C T
Habitat destruction, often caused by anthropogenic disturbance, can lead to the extinction of species at an unprecedented rate. It is important, therefore, to consider habitat destruction when assessing population viability. Another factor often ignored in population viability analysis, is the Allee effect that adds to the risk of populations already on the verge of extinction. Understanding the Allee effect on species dynamics and response to habitat destruction has intrinsic value in conservation prioritization. Here, the Allee effect was considered in a multi-species hierarchical competition model. Results showed that species persistence declines dramatically due to the Allee effect, and certain species become more susceptible to habitat destruction than others. Two extinction orders emerged under habitat destruction: either the best competitor becomes extinct first or the best colonizer first. The extinction debt and order, as well as the time lag between habitat destruction and species extinction, were found to be determined by species abundance and the intensity of the Allee effect.
\end{abstract}

Crown Copyright $\odot 2009$ Published by Elsevier Inc. All rights reserved.

\section{Introduction}

The persistence of natural populations faces three challenges: habitat destruction, climatic change and biological invasion, which are all human associated [1]. Studies have shown that species disappear up to 1000 times faster than without anthropogenic disturbance [2]. Among these challenges, habitat destruction has been suggested to be the foremost cause of species extinction [3-5]. Consequently, understanding the dynamics of extinction, as well as species' response to the habitat destruction, is crucial for conservation planning and management [6-14].

An important concept in this domain is the extinction debt, defined as the number of future species loss by current habitat destruction [15-24]. In particular, Tilman et al. [7], using a hierarchical competition model, generalized an earlier study of Nee and May [25] in examining the effect of habitat destruction through the patch-removal test. Two important conclusions from their study are: (1) the best competitor becomes extinct first, and (2) habitat destruction leads to the extinction debt (a delayed extinction of certain species). However, these two conclusions have been questioned due to the oversimplification of Tilman et al.'s model [17,26-28]. For instance, it is possible for a species other than the best competitor to become extinct first if its colonization rate satisfies certain conditions [9]. Nonetheless, these studies all conclude that, at least, the worst competitor (i.e., the best colonizer

\footnotetext{
* Corresponding author.

E-mail addresses: cllha@163.com (L. Chen), chui@sun.ac.za (C. Hui).
}

due to the colonization-competition trade-off), will not be the first one driven to extinction by habitat destruction.

Even though the extinction debt and species extinction order (time sequence) are of paramount value for understanding species succession dynamics and implementing efficient conservation plans, its impact on rare species (or populations on the verge of extinction) is often underestimated. Rare species can become extinct through the Allee effect [29], which describes a positive correlation between any measures of species fitness and population size [30-32]. The Allee effect is closely related to the population viability, and has been shown to play an important role in conservation prioritization [33,34]. Recent modeling progress has allowed the consequences of the Allee effect to be revealed in a wide range of ecological questions, such as the spread of invasive species [35-38], the optimal distance of dispersal [39], invasion pinning [40], the spatiotemporal dynamics of metapopulations [41-43] and the stability of competing populations [44]. As a result, the influence of the Allee effect on species extinction order under habitat destruction and extinction debt is worthy of consideration.

The Allee effect can be classified into weak and strong forms [34], depending on the opposing strengths of the positive (Allee effect mechanism) and negative density dependence (intraspecific competition). The population is facing a strong Allee effect if the growth rate is negative when the population size is low. The population size at which the growth rate becomes negative is coined the Allee effect threshold. If the population size is below this threshold, the species will become extinct. When the growth rate remains positive at low population size, the Allee effect is called 
weak. No threshold of the population size exists for the weak Allee effect. Here, we test the influence of the Allee effect on extinction debt and species extinction order. Specifically, three issues are examined under the habitat destruction with and without Allee effects: (1) the dynamics and equilibria of the hieratically competition model of metapopulations, (2) the formulae of the extinction debt and species extinction order, and (3) the time lag between habitat destruction and species extinction.

\section{Model}

Tilman et al.'s [7] hierarchical competition model assumes a homogenous landscape divided into cells (patches or lattices) with a size that can only host one individual (or one local population as in metapopulations), i.e., an individual-based model. Population persistence is balanced between the mortality of individuals and the colonization of empty cells. Species that are superior in competition suffer a low dispersal (colonization) capacity (rate). The multi-species coexistence maintains through the colonizationcompetition trade-off of species life-history characteristics [45]. The model is defined in terms of the proportion of cells occupied by species $i$ ( $p_{i}$; called either the population abundance or occupancy), species-specific colonization rate $\left(m_{i}\right)$ and mortality (or local extinction) rate $\left(e_{i}\right)$, and the amount (proportion) of destroyed habitat $(D)$. Superior competitors, once successful colonizing a cell, can instantly displace inferior ones from the cell. This hierarchical competition model can be written as follows,

$\frac{d p_{i}}{d t}=m_{i} p_{i}\left(1-D-\sum_{j=1}^{i} p_{j}\right)-e_{i} p_{i}-\sum_{j=1}^{i-1} p_{i} m_{j} p_{j}$.

The three terms on the right represent population recruitment through colonizing empty cells, the independent mortality, and the mortality due to competitive displacement, respectively. At equilibrium the population abundance of the $i$ th species is

$p_{i}^{*}=1-D-\frac{e_{i}}{m_{i}}-\sum_{j=1}^{i-1} p_{j}^{*}\left(1+\frac{m_{j}}{m_{i}}\right)$.

Note that this must be solved from species 1 (the best competitor) to species $i\left(m_{i}>0\right.$ and $p_{i}^{*} \geqslant 0$ for all $\left.i\right)$. Here, we applied the same parameter values as in Tilman et al. $[7,8]$ in order to compare with their results; that is, population abundance at equilibrium forms a geometric series, $p_{i}^{0}=q(1-q)^{i-1}$, where $q$ is the abundance of the best competitor. Under the same mortality $(e)$ among species, the equilibrium also infers a geometric form of the colonization rate, $m_{i}=e /(1-q)^{2 i-1}$. The position of a species in the sequence of extinction is determined by the ranking of its abundance at equilibrium when no other species exist in the community, $\hat{p}_{i}=$ $1-e_{i} / m_{i}$. Tilman et al. [7,8] applied model (1) and above parameters to analyze the effect of habitat destruction on the dynamics simulating a temperate forest $(q=0.2)$ and a tropical forest ( $q=0.03$ ), with each community including 20 species. Due to the demographic and environmental stochasticity, we consider species with its density less than $10^{-6}$ extinction. To examine a community with $k$ species, we hereafter simply let $p_{k}^{0}=q(1-q)^{k-1}=10^{-6}$ and $k=1-(6+\log q) /(\log [1-q])$.

Nee and May [25] found that once a portion of habitat equal to the equilibrium of the focal species is destroyed, the species is doomed to extinction ( $p_{1}^{*}=0$ if $D \geqslant 1-e_{1} / m_{1}$; see Fig. 1 ). For instance, after substituting $m_{i}$ and $e_{i}$ into Eq. (2), we have the proportion of habitat loss that can cause the extinction of $i$ th species, $D_{i} \geqslant 1-(1-q)^{2 i-1}$. Because $D_{1}<D_{2}<D_{3}<\ldots$, species become extinct sequentially from the best to the worst competitor as the habitat destruction increases. The amount of habitat destruction sufficient to cause the extinction of the $i$ th species is



Fig. 1. Population abundance for the best competitor as a function of the colonization rate $m_{1}$ and the fraction of habitat destroyed $D$ according to the stable equilibrium $p_{1}^{*}$ with $e_{1}=0.2$.

$D_{i}=1-(1-q)^{2 i-1}$

Furthermore, the deterministic extinction of species, from the best to the worst competitor, does not follow habitat destruction immediately but is delayed. Such delayed extinction is analogous to a debt caused by current habitat destruction that will be paid by certain species in the future (i.e., a predetermined future). This extinction debt $(E)$ measures the total number of species driven to extinction at equilibrium by a given amount of habitat destruction $(D)$. Substituting $E$ for $i$ in Eq. (3), we have the number of species driven to extinction by habitat destruction,

$E=\frac{\ln [(1-D)(1-q)]}{2 \ln [1-q]}$.

The above dynamics and the extinction debt can be largely altered by Allee effect. The dynamics of multiple species coexisting in a metapopulation community subject to an Allee effect can be given as follows,

$$
\begin{aligned}
\frac{d p_{i}}{d t}= & m_{i} p_{i}\left(1-D-\sum_{j=1}^{i} p_{j}\right)\left(\frac{p_{i}-a A_{i}}{p_{i}+b A_{i}}\right)-e_{i} p_{i} \\
& -\sum_{j=1}^{i-1} p_{i} m_{j} p_{j}\left(\frac{p_{j}-a A_{j}}{p_{j}+b A_{j}}\right),
\end{aligned}
$$

where $\left(p_{i}-a A_{i}\right) /\left(p_{i}+b A_{i}\right)$ denotes the species-specific Allee effect, which assumingly only affects the colonization process. This is because mortality is often considered independent in metapopulation models [27] and therefore does not suffer from the Allee effect. Clearly, Eq. (5) is a generalization of Eq. (1) $\left(A_{i}=0\right)$. Windus and Jensen [24] also analyzed similar metapopulation models subject to an Allee effect that only affects the colonization process. Parameter $A_{i}$ can be defined as the intensity of Allee effect of species $i$; coefficients $a$ and $b$ are two constants that determine the forms (weak/strong) of Allee effect. Eq. (5) has two equilibria,

$p_{i}^{*}=\frac{1}{2}\left(X+a A_{i}-Y \pm \sqrt{\left(X+a A_{i}-Y\right)^{2}-4 A_{i}(a X+b Y)}\right)$,

where $X=\left(1-D-\sum_{j=1}^{i-1} p_{j}^{*}\right)$ and $Y=\left(e_{i} / m_{i}\right)+\sum_{j=1}^{i-1} p_{j}^{*} m_{j}\left(p_{j}^{*}-a A_{j}\right) /$ $\left(m_{i}\left(p_{j}^{*}+b A_{j}\right)\right)$. If the coefficient $a$ is species specific, $a_{i}=-b \cdot e_{i} /$ $m_{i}=-b \cdot(1-q)^{2 i-1}$, all species undergo a weak Allee effect. This weak Allee effect implies that the species only has one positive 
equilibrium and suffers from a low per capita growth rate $(d p / d t)(1 / p) \approx 0$ when the population abundance is low $(p \approx 0)$ (Fig. 2). We here, however, focused only on the strong Allee effect. The weak Allee effect can be analyzed using a similar procedure as outlined below.

We divided the strong Allee effect into weak and strong forms affecting the colonization process (Fig. 2). For convenience, Alleelike effect hereafter only infers the one for the per capita colonization rate, distinguished from the traditional definition of Allee effect for the per capita growth rate. If $a=0, b \in(0,1]$, species subjects to a weak Allee-like effect (without a threshold for the colonization rate) (Fig. 2). If $a \in(0,1], b=0$, species undergoes a strong Allee-like effect with a threshold for the colonization rate, $a A_{i}$, below which species cannot further colonize empty patches (Fig. 2). The two equilibria of the system under these two scenarios can be solved by Eq. (6) from species 1 (superior competitor) to less competitive species under conditions $m_{i}>0$ and $p_{i}^{*} \geqslant 0$.

\section{Results}

\subsection{Weak Allee-like effect}

The two equilibria of the best competitor subject to a weak Allee-like effect for colonization process, under additional conditions $e_{1}<m_{1}(1-D)$ and $\left(1-D-e_{1} / m_{1}\right)^{2} \geqslant 4 b A_{1} e_{1} / m_{1}$, are,

$p_{11}^{*}=\frac{1}{2}\left(1-D-\frac{e_{1}}{m_{1}}+\sqrt{\left(1-D-\frac{e_{1}}{m_{1}}\right)^{2}-4 b A_{1} \frac{e_{1}}{m_{1}}}\right)$

$p_{12}^{*}=\frac{1}{2}\left(1-D-\frac{e_{1}}{m_{1}}-\sqrt{\left(1-D-\frac{e_{1}}{m_{1}}\right)^{2}-4 b A_{1} \frac{e_{1}}{m_{1}}}\right)$

This discontinuity in the solution is due to the existence of a threshold (dashed line in Fig. 3). If there is no habitat destruction $(D=0)$, the population abundance $(p)$ subject to a weak Allee-like effect ( $B$ in Fig. 3 ) is less than predicted from the Tilman model

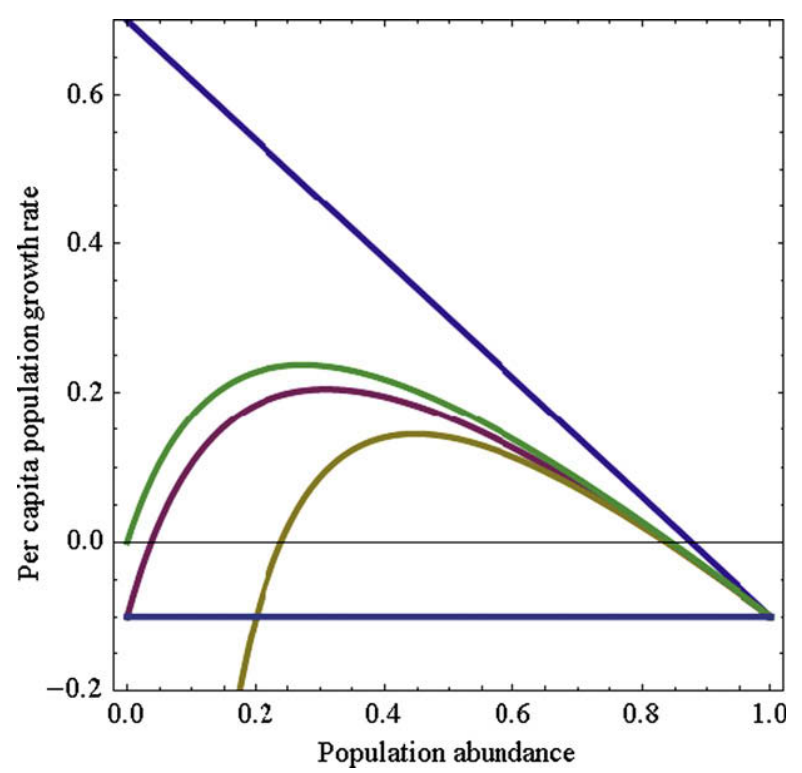

Fig. 2. Relationships between the per capita population growth rate and the population abundance for the negative density dependence (blue line), the weak Allee effect (green curve; $a=-b \cdot e / m$ ), weak (red curve; $a=0.4$ ) and strong (brown curve; $b=0.5$ ) Allee-like effects for colonization process. Two flat lines indicate zero per capita growth rate and the minus extinction rate $(e=0.1)$. Other parameters: $m=0.8$ and $A=0.5$.

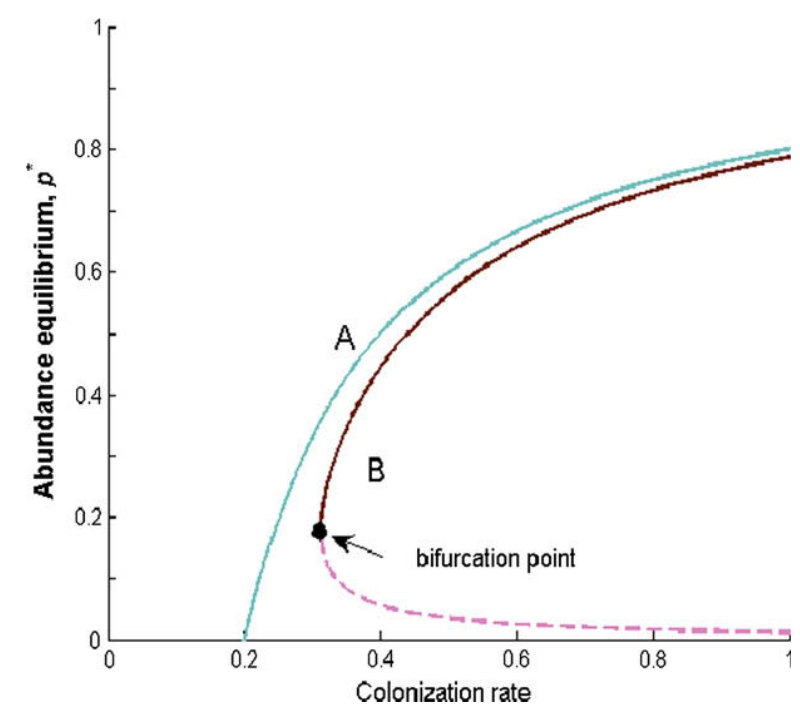

Fig. 3. Alternative equilibria due to the weak Allee effect. Continuous lines depict stable equilibria; dashed lines unstable equilibrium. Letters A and B stand for the basic Tilman model without Allee effect and the model subject to the weak Allee effect, respectively. Parameters: $e_{1}=0.2, b=0.25$ and $A_{1}=0.2$.

(A in Fig. 3). The Allee-like effect leads to alternative equilibria separated by the dashed line as a watershed threshold (Fig. 3). If the initial population abundance is above the unstable equilibrium $p_{12}^{*}$, the population will converge upwards to the stable equilibrium $p_{11}^{*}$; otherwise, the population will become extinct.

Even without habitat destruction, species can still become deterministically extinct if the weak Allee-like effect reaches its maximum value (Fig. 4A),

$b A_{1} \leqslant \frac{m_{1}\left(1-e_{1} / m_{1}\right)^{2}}{4 e_{1}}$,

where $e_{1}<m_{1}$. The stronger the Allee-like effect is, the less population abundance remains and, therefore, the higher the colonization rate that is required to support population persistence. When a portion of $D$ habitat is permanently destroyed (Fig. 4B), the species undergoes deterministic extinction once the critical value $D_{c}$ is reached,

$D_{c}=1-\frac{e_{1}}{m_{1}}-\sqrt{4 b A_{1} \frac{e_{1}}{m_{1}}}$.

That is, the higher the intensity of Allee-like effect $A_{1}$ is, the less habitat can be destroyed before the population becomes extinct.

Under the weak Allee-like effect, the stable equilibrium for a single-species system is given by Eq. (7). The species can survive only if $D_{i} \leqslant 1-\left(e_{i} / m_{i}\right)-\sqrt{4 b A_{i} e_{i} / m_{i}}$. Consequently, the $i$ th species will become extinct if habitat destruction reaches the threshold,

$D_{i}=1-(1-q)^{2 i-1}-\sqrt{4 b A_{i}(1-q)^{2 i-1}}$.

This result revealed the uncertainty of species extinction order due to species-specific intensity of Allee-like effect. If $A_{i-1}>A_{i}(1-q)^{2}$, species becomes extinct in the same order as its competitive ranking because of $D_{i-1}<D_{i}$. Therefore, the extinction debt $E$ is equal to the serial number of species $i$,

$E=\frac{\ln \left[(1-q)\left(1-D+2\left(b A+\sqrt{(b A)^{2}+b A-b A D}\right)\right)\right]}{2 \ln (1-q)}$.

When the best competitor is rare ( $q=0.1$; the green line in Fig. 5$)$, the extinction debt increases rapidly with habitat loss, faster than when the best competitor is relatively common ( $q=0.2$; the yellow 

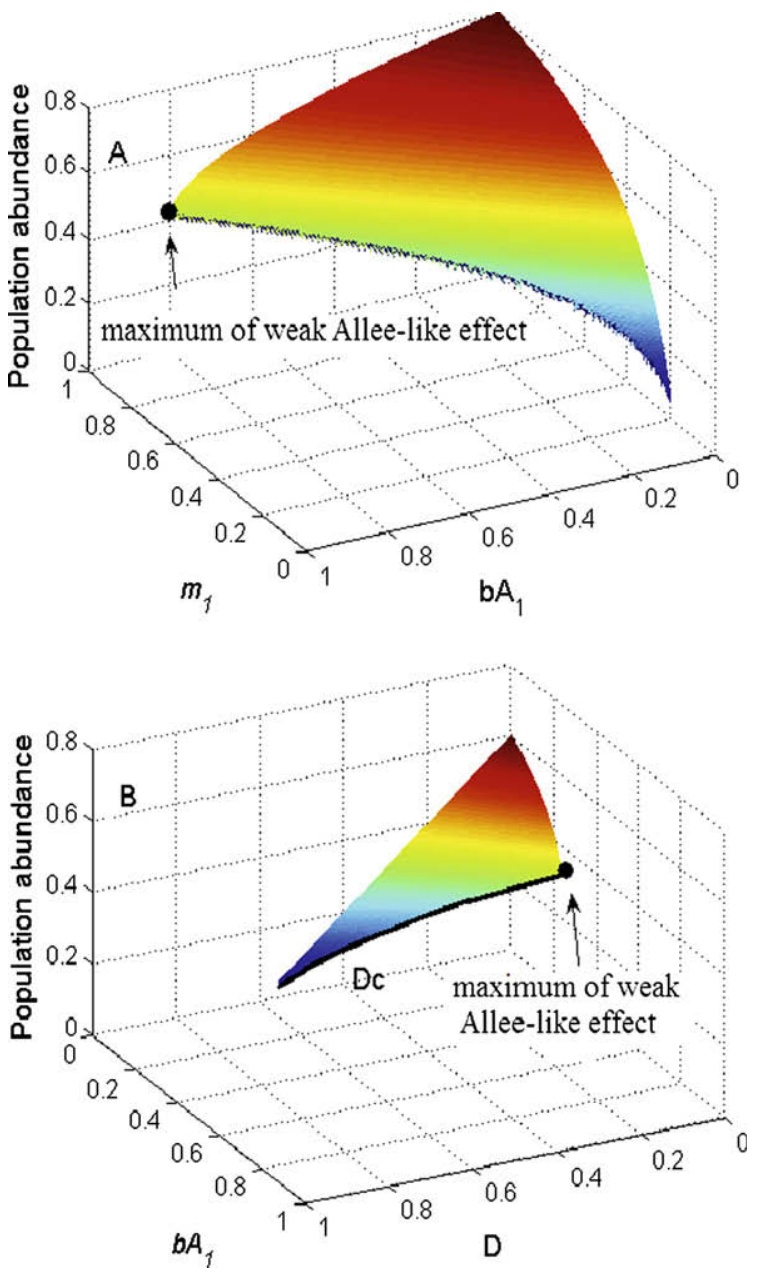

Fig. 4. The response of stable equilibrium to a weak Allee effect according to the stable equilibrium $p_{11}^{*}$ (Eq. (7)). (A) Population abundance is a function of the colonization rate $m_{1}$ and the weak Allee effect $b A_{1}$. (B) Population abundance is a function of the fraction of habitat destroyed $D$ and the weak Allee effect $b A_{1}$. Parameters: $e_{1}=0.2$ for (A) and (B); $m_{1}=0.5$ for (B).

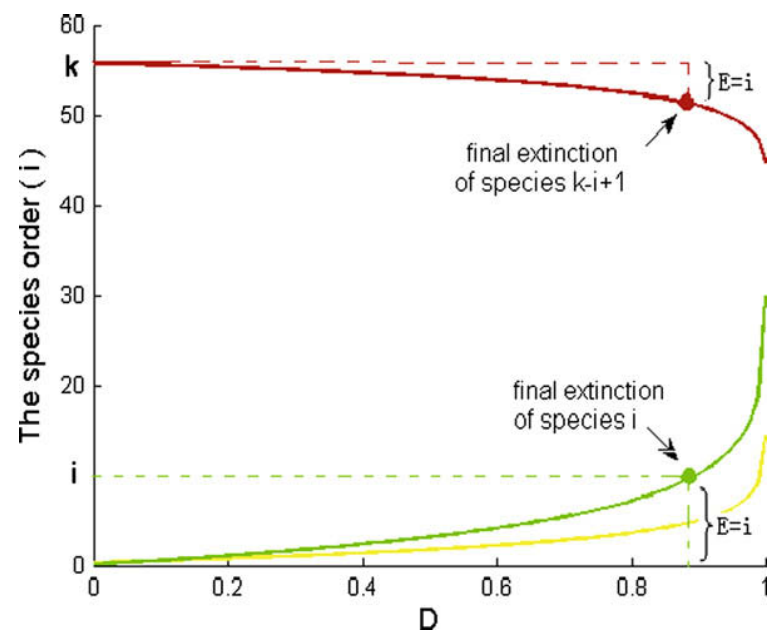

Fig. 5. An illustration of the relationship between species extinction order and the habitat destruction in the model subject to a weak Allee effect. The yellow and green lines depict the scenario that better competitors are driven extinct first; the red line indicates the scenario that better colonizers become extinct first. (For interpretation of the references to color in this figure legend, the reader is referred to the web version of this article.) line in Fig. 5). If $(1-q)^{2 i-3}+\sqrt{4 b A_{i-1}(1-q)^{2 i-3}}<(1-q)^{2 i-1}+$ $\sqrt{4 b A_{i}(1-q)^{2 i-1}}$, the species are driven extinct in the order starting from the worst competitor because of $D_{i-1}>D_{i}$. Therefore, in a $k$ species community, the species $k$ will be first extinct and the species $k-i+1$ last ( $q=0.2$; the red line in Fig. 5 ). The extinction debt $E$ has the same form as Eq. (12).

\subsection{Strong Allee-like effect}

In a hierarchal competition model subject to a strong Allee-like effect in the colonization process, two equilibria of the best competitor ( $p_{11}^{*}$ stable; $p_{12}^{*}$ unstable) are as follows,

$$
p_{11}^{*}=\frac{1}{2}\left(1-D+a A_{1}-\frac{e_{1}}{m_{1}}+\sqrt{\left(1-D+a A_{1}-\frac{e_{1}}{m_{1}}\right)^{2}-4 a A_{1}(1-D)}\right)
$$

$p_{11}^{*}=\frac{1}{2}\left(1-D+a A_{1}-\frac{e_{1}}{m_{1}}-\sqrt{\left(1-D+a A_{1}-\frac{e_{1}}{m_{1}}\right)^{2}-4 a A_{1}(1-D)}\right)$,

under additional conditions $\left(1-D+a A_{1}\right) \geqslant e_{1} / m_{1}$ and $(1-D-$ $\left.a A_{1}-e_{1} / m_{1}\right)^{2} \geqslant 4 a A_{1}(1-D)$. As shown in Fig. $6 \mathrm{~A}$, the range of
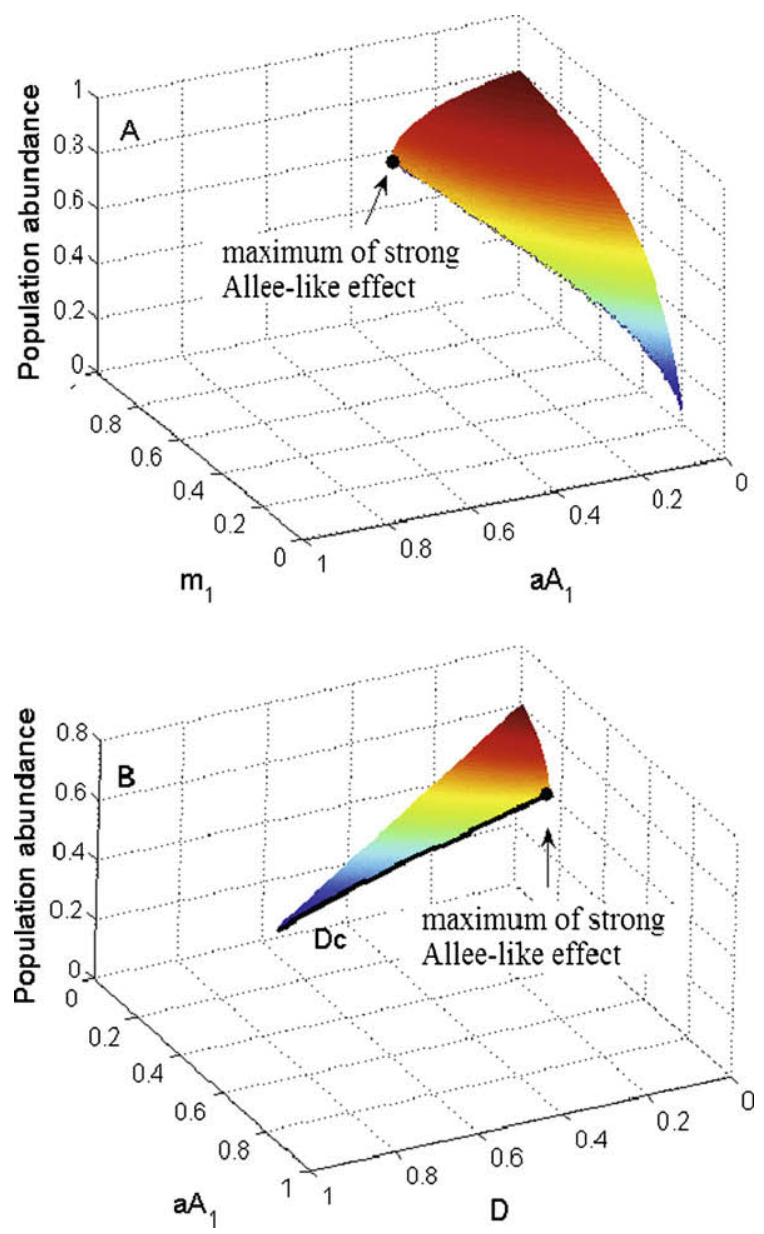

Fig. 6. The response of stable equilibrium to a strong Allee effect according to the stable equilibrium $p_{11}^{*}$ (Eq. (13)). (A) Population abundance is a function of the colonization rate $m_{1}$ and the strong Allee effect $a A_{1}$. (B) Population abundance is a function of the fraction of habitat destroyed $D$ and the strong Allee effect $a A_{1}$. Parameters are the same as in Fig. 4. 
colonization rate, for population persistence, decreases sharply as the intensity of Allee effect increases. Even without habitat destruction $(D=0)$, species becomes extinct if $a A_{1}>1-e_{1} /$ $m_{1}-\sqrt{4 e_{1} / m_{1}}$. The threshold of habitat destruction is as follows,

$D_{c}=1-\frac{e_{1}}{m_{1}}-a A_{1}-2 \sqrt{a A_{1} \frac{e_{1}}{m_{1}}}$.

Even a small amount of habitat destruction can lead to the elimination of a large number of species (Fig. 6B).

When the single-species system suffers from a strong Allee effect, the stable equilibrium of population abundance is given by Eq. (13). If a fraction of habitat is destroyed, the species can survive only if $D_{i} \leqslant 1-\left(e_{i} / m_{i}\right)-a A_{i}-2 \sqrt{a A_{i} e_{i} / m_{i}}$, and therefore the extinction threshold of the $i$ th species is given by

$D_{i}=1-(1-q)^{2 i-1}-a A_{i}-2 \sqrt{a A_{i}(1-q)^{2 i-1}}$.

This also implies two extinction orders. If $D_{i-1}<D_{i}$, species will become extinct sequentially according to the competition ranking; otherwise, the extinction order will reverse. The extinction debt can be obtained as follows,

$E=\frac{\ln [(1-q)(1-D+a A+2 \sqrt{a A(1-D)})]}{2 \ln (1-q)}$.
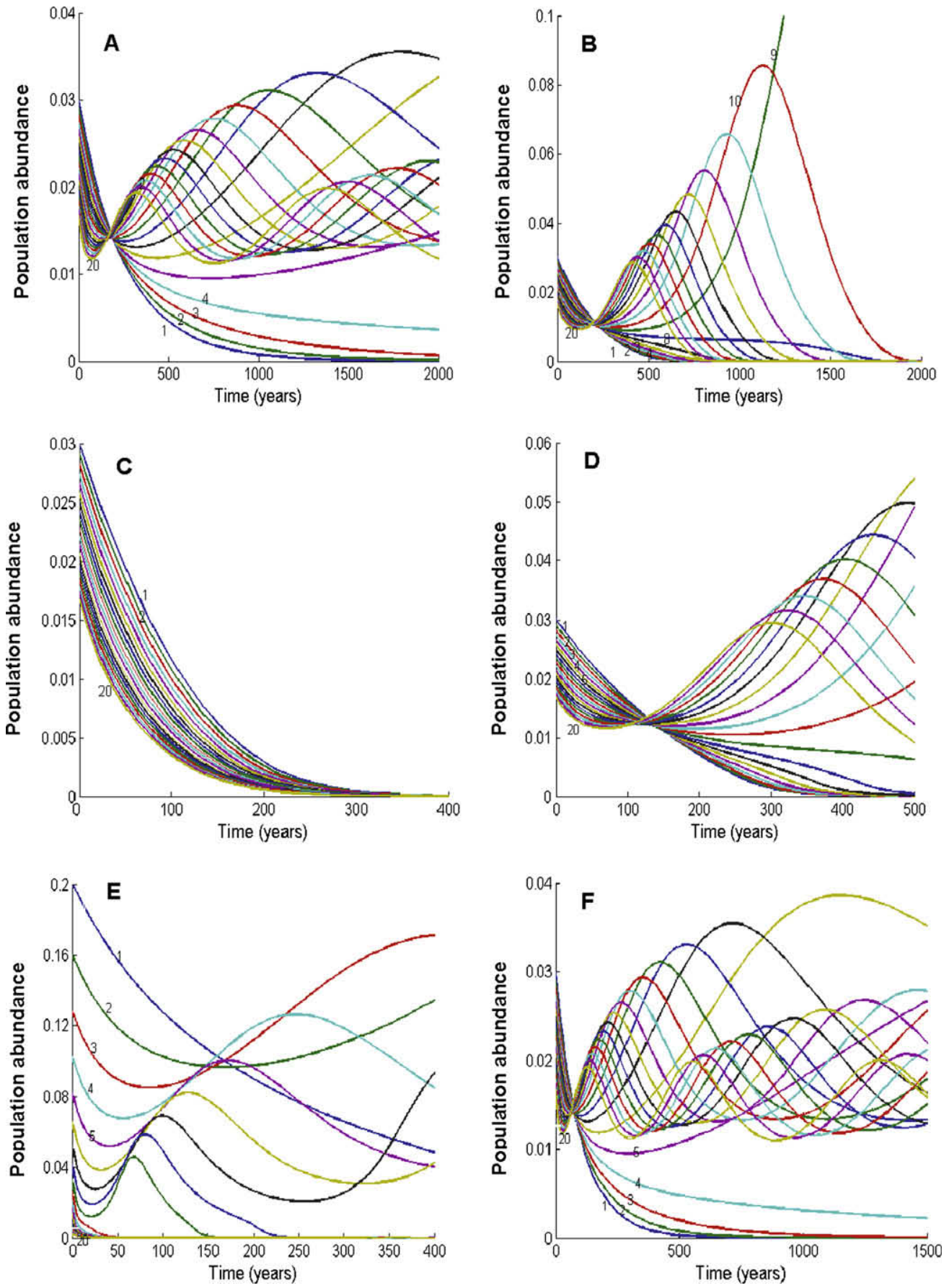

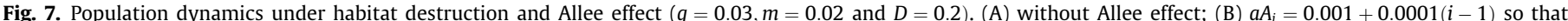

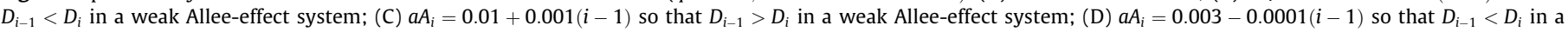
strong Allee-effect system; (E) $q=0.2$ and $a A_{i}=0.005$ so that $D_{i-1}>D_{i}$ in a strong Allee-effect system; (F) $m=0.05$ without Allee effect. 


\subsection{The time-lag}

All the above results only apply when the system is in equilibrium. However, the model also predicts a time delay between habitat destruction and the follow-on extinction. Considering 20 coexisting species that experience a $20 \%$ habitat loss, we expect the extinction of the dominant species. However, it took more than 1000 time steps (years) for the dominant species to become extinct when using Tilman's parameters (Fig. 7A). In a system subject to Allee effects, the above equilibrium model can correctly identify which and how many species go extinct by habitat destruction. In the system with a weak Allee effect, as shown in Fig. 7B, a time lag of 700-900 years was found following the habitat destruction if $A_{i-1}>A_{i}(1-q)^{2}$ (i.e., the best competitor becomes extinct first); and a time lag of less than 400 years was found if $(1-q)^{2 i-3}+$ $\sqrt{4 b A_{i-1}(1-q)^{2 i-3}}<(1-q)^{2 i-1}+\sqrt{4 b A_{i}(1-q)^{2 i-1}} \quad$ (Fig. 7C; the worst competitor becomes extinct first). Analogous to the weak Allee system, a time lag of 400-500 years (Fig. 7D) and less than 50 years (Fig. 7E) occurred for extinction orders starting from the best and worst competitor, respectively. Furthermore, if we increase the mortality of the population (Fig. 7F), only a subtle difference is found compared to Fig. 7A. Simply increasing mortality will not change the order of extinction, but only speed up the extinction dynamics, leading to a shorter time-lag. Therefore, the mortality rate should be considered in the calculation of the time lag before the extinction debt is paid.

\section{Discussion}

Different models have been designed to tackle the question of species extinction, especially the extinction order and debt caused by habitat destruction. Following Nee and May's [25] argument, Tilman et al. $[7,8]$ warned that the superior species (best competitor) will become extinct first in both tropical and temperate forests after a time lag of 50-400 years between habitat destruction and the extinction of dominant competitors. Dytham [26] has used a cellular automaton to reexamine the effect of habitat destruction on competitive coexistence in Nee and May's (1992) model, so has Moilanen and Hanski [17] using a spatially realistic incidencefunction model. Both studies suggest the basic assumptions in Nee and May [25] to be utter simplifications of the reality, which makes the model an unsound basis for conservation planning. Malanson [46] reached a conclusion on the impact of per unit habitat loss: better competitors suffer more than poor competitors when the habitat loss is low, whereas better colonizers suffer more than poor colonizers when the habitat loss is high. Using numerical simulations, Hanski and Ovaskainen [19] showed that extinction debt is especially high in a community with most species on the verge of extinction. Our result suggests, however, that only considering a limited number of time steps in simulations and ignoring time delays in population dynamics can lead to an underestimation of the extinction debt and the number of endangered species under habitat destruction.

Apart from habitat destruction, the Allee effect has also been widely recognized as an important threat to the survival of rare species $[41,42,47,48]$. Various mechanisms have been proposed as the potential sources of Allee effect, such as the need of a minimal number of individuals necessary to successfully produce and raise offspring, forage, anti-predation, and avoid genetic inbreeding [31,49-51]. Using a phase-plane analysis, Zhou et al. [52] showed that the species susceptible to the Allee-like effect will be more susceptible to habitat destruction. Windus and Jensen [24] used a lattice-based model and found that there exists a critical population density below which the probability of extinction is greatly increased due to Allee effect. Our results emphasize the combined effect of both the Allee effect and habitat destruction.

In this paper, we introduced two types of Allee effects, i.e., weak and strong Allee effects (departing somewhat from the traditional definition), in Tilman's multi-species competitive model to explore the extinction events caused by habitat destruction. First, Allee effect causes a fundamental alteration of the phase plane of the model. Habitat destruction can only lead to a single stable equilibrium in the system when there is no Allee effect $[45,53]$, but two equilibria exists, one stable and the other unstable, when populations are subject to the Allee effect. The stronger the Allee effect is, the more difficult is for the species to sustain itself (Figs. 4 and 6A), which makes the species more susceptible to habitat destruction (Figs. 4 and 6B). Furthermore, compared to the weak Allee effect, the strong Allee effect can drastically shorten the time delay of species extinction, resulting in a much more sudden extinction event.

The extinction order and debt are revised, depending on the abundance of the best competitor $(q)$ and the intensity of the Allee effect $\left(A_{i}\right)$. Two extinction scenarios emerged: the best competitor goes extinct first, or the best colonizer goes extinct first. Interestingly, both scenarios have the same extinction debt. It suggests that Tilman et al.'s [7] conclusion is a special case of our model. The inferior competitor may become the first victim following habitat destruction, due solely to the Allee effect. Moreover, the time lag in Tilman's model might be overestimated because of the overwhelming influence of Allee effects in ecological systems [54]. It is important to notice that the time lag varies between weak and strong Allee effects and is entangled in a complicated relationship between $q$ and $A_{i}$. Moreover, besides these two variables in determining the extinction debt and time lag, species mortality $m$ should also be included especially in the calculation of the time lag of extinction after habitat destruction (Fig. 7).

In conclusion, the extinction debt caused by habitat destruction is not only paid by better competitors in the community. The Allee effect, colonization-competition trade-off and species abundance patterns, together, determine the extinction debt and order. Besides species with superior competition ability, species with strong colonization ability but which are particularly susceptible to the Allee effect should also be prioritized in conservation planning.

\section{Acknowledgments}

We are grateful to Laura C. Reed, Beverley Laniewski and two anonymous referees for constructive comments. C.H. also acknowledges the support from the Blue Skies Programme of the National Research Foundation and the DST-NRF Centre of Excellence for Invasion Biology.

\section{References}

[1] R. Barbault, S.D. Sastrapradja, Generation, maintenance and loss of biodiversity, in: W.H. Heywood (Ed.), Global Biodiversity Assessment, Cambridge University Press, Cambridge, 1995, p. 193.

[2] H.Y. Liu, Z.S. Lin, T. Wen, Responses of metapopulation dynamics to two different kinds of habitat destruction caused by human activities, Plant Ecol. 188 (2007) 53.

[3] L. Fahrig, Relative effects of habitat loss and fragmentation on population extinction, J. Wildlife Manage. 61 (1997) 603

[4] S.L. Pimm, P. Raven, Extinction by numbers, Nature 403 (2000) 843-845.

[5] A.M. Rosser, S.A. Mainka, Overexploitation and species extinctions, Cons. Biol. 16 (2002) 584

[6] P. Ehrlich, A. Ehrlich, Extinction: The Causes and Consequence of the Disappearance of Species, Random House, New York, 1981.

[7] D. Tilman, R.M. May, C.L. Lehman, M.A. Nowak, Habitat destruction and the extinction debt, Nature 371 (1994) 65.

[8] D. Tilman, C.L. Lehman, C. Yin, Habitat destruction, dispersal, and deterministic extinction in competitive communities, Am. Nat. 149 (1997) 407.

[9] C.A. Klausmeier, Extinction in multispecies and spatially explicit models of habitat destruction, Am. Nat. 152 (1998) 303.

[10] G. Ceballos, P.R. Ehrlich, Mammal population losses and the extinction crisis, Science 296 (2002) 904. 
[11] O. Ovaskainen, I. Hanski, Spatially structured metapopulation models: global and local assessment of metapopulation capacity, Theor. Popul. Biol. 60 (2001) 218 .

[12] O. Ovaskainen, K. Sato, J. Bascompte, I. Hanski, Metapopulation models for extinction threshold in spatially correlated landscapes, J. Theor. Biol. 215 (2002) 95.

[13] J. Bascompte, Extinction thresholds: insights from simple models, Ann. Zool. Fenn. 40 (2003) 99.

[14] T.G. Benton, Understanding the ecology of extinction: are we close to the critical threshold?, Ann Zool. Fenn. 40 (2003) 71.

[15] R. Levins, Some demographic and genetic consequences of environmental heterogeneity for biological control, Bull. Entomol. Soc. Am. 15 (1969) 237.

[16] R.M. May, M.A. Nowak, Superinfection, metapopulation dynamics, and the evolution of diversity, J. Theor. Biol. 170 (1994) 95.

[17] A. Moilanen, I. Hanski, Habitat destruction and coexistence of competitors in a spatially realistic metapopulation model, J. Anim. Ecol. 64 (1995) 141.

[18] L. Fahrig, Effect of habitat fragmentation on the extinction threshold: a synthesis, Ecol. Appl. 12 (2002) 346.

[19] I. Hanski, O. Ovaskainen, Extinction debt at extinction threshold, Cons. Biol. 16 (2002) 666.

[20] Z.S. Lin, Z.L. Xie, Does habitat restoration cause species extinction?, Biol Cons. 123 (2004) 349.

[21] Z.S. Lin, The ecological order of persisting species during habitat destruction, Ecol. Model. 184 (2005) 249.

[22] A. Baldi, J. Voros, Extinction debt of Hungarian reserves: a historical perspective, Basic Appl. Ecol. 7 (2006) 289.

[23] C.R. Bulman, R.J. Wilson, A.R. Holt, L.G. Bravo, R.I. Early, M.S. Warren, C.D. Thomas, Minimum viable metapopulation size, extinction debt, and the conservation of a declining species, Ecol. Appl. 17 (2007) 1460.

[24] A. Windus, H.J. Jensen, Allee effects and extinction in a lattice model, Theor. Popul. Biol. 72 (2007) 459.

[25] S. Nee, R.M. May, Dynamics of metapopulations: habitat destruction and competitive coexistence, J. Anim. Ecol. 61 (1992) 37.

[26] C. Dytham, Habitat destruction and competitive coexistence: a cellular model, J. Anim. Ecol. 63 (1994) 191.

[27] I. Hanski, A practical model of metapopulation dynamics, J. Anim. Ecol. 63 (1994) 151.

[28] C. Loehle, B.L. Li, Habitat destruction and the extinction debt revisited, Ecol. Appl. 6 (1996) 784.

[29] W.C. Allee, Animal Aggregations: A Study in General Sociology, University of Chicago Press, Chicago, 1931.

[30] F. Courchamp, B. Grenfell, B.T. Clutton, Population dynamics of obligate cooperators, Proc. R. Soc. Lond. B 266 (1999) 557-563.

[31] P.A. Stephens, W.J. Sutherland, Consequences of the Allee effects for behaviour, ecology and conservation, Trends Ecol. Evol. 14 (1999) 401.

[32] P.A. Stephens, W.J. Sutherland, R.P. Freckleton, What is the Allee effect?, Oikos 87 (1999) 185

[33] D.S. Boukal, L. Berec, Single-species models of the Allee effects: extinction boundaries, sex ratios and mate encounters, J. Theor. Biol. 218 (2002) 375.
[34] L. Berec, E. Angulo, F. Courchamp, Multiple Allee effects and population management, Trends Ecol. Evol. 22 (2007) 185.

[35] M.A. Lewis, P. Kareiva, Allee dynamics and the spread of invading organisms, Theor. Popul. Biol. 43 (1993) 141.

[36] L. Berec, D.S. Boukal, M. Berec, Linking the Allee effect sexual reproduction and temperature-dependent sex determination via spatial dynamics, Am. Nat. 157 (2001) 217.

[37] M.H. Wang, M. Kot, M.G. Neubert, Integrodifference equations, Allee effects and invasions, J. Math. Biol. 44 (2002) 150

[38] A.S. Ackleh, L.J.S. Allen, J. Carter, Establishing a beachhead: a stochastic population model with an Allee effect applied to species invasion, Theor Popul. Biol. 71 (2007) 290.

[39] A.B. South, R.E. Kenward, Mate finding, dispersal distances and population growth in invading species: a spatially explicit model, Oikos 95 (2001) 53.

[40] T.H. Keitt, M.A. Lewis, R.D. Holt, Allee effects invasion pinning and species' borders, Am. Nat. 157 (2001) 203.

[41] P. Amarasekare, Allee effects in metapopulation dynamics, Am. Nat. 152 (1998) 298.

[42] C.E. Brassil, Mean time to extinction of a metapopulation with an Allee effect, Ecol. Model. 143 (2001) 9.

[43] C. Hui, Z.Z. Li, Distribution patterns of metapopulation determined by Allee effects, Popul. Ecol. 46 (2004) 55.

[44] G. Wang, X.G. Liang, F.Z. Wang, The competitive dynamics of populations subject to an Allee effect, Ecol. Model. 124 (1999) 183

[45] D. Tilman, Competition and biodiversity in spatially structured habitats, Ecology 75 (1994) 2

[46] G.P. Malanson, Extinction-debt trajectories and spatial patterns of habitat destruction, Ann. Assoc. Am. Geogr. 92 (2002) 177.

[47] R.H. Lamberson, R. McKelvey, B.N. Noon, C. Voss, A dynamic analysis of northern spotted owl viability in a fragmented forest landscape, Cons. Biol. 6 (1992) 505.

[48] M.J. Groom, Allee effects limit population viability of an annual plant, Am. Nat 151 (1998) 487.

[49] J. Jacobs, Cooperation, optimal density and low density threshold: yet another modification of the logistic model, Oecologia 64 (1984) 389.

[50] B.E. Saether, T.H. Ringsby, E. Roskaft, Life history variation, population processes and priorities in species conservation: towards a reunion of research paradigms, Oikos 77 (1996) 217.

[51] A.P. Moller, S. Legendre, Allee effect, sexual selection and demographic stochasticity, Oikos 92 (2001) 27.

[52] S.R. Zhou, C.Z. Liu, G. Wang, The competitive dynamics of metapopulation subject to the Allee-like effect, Theor. Popul. Biol. 65 (2004) 29

[53] R. Levins, Extinction, in: M. Gerrstenhaber (Ed.), Lectures on Mathematics in the Life Sciences, vol. 2, American Mathematical Society, Providence, 1970, p. 77.

[54] F. Courchamp, L. Berec, J. Gascoigne, Allee Effects in Ecology and Conservation, Oxford University Press, Oxford, 2008. 